\title{
Priming of Control: Implicit Contextual Cuing of Top-down Attentional Set
}

\author{
Joseph A. King, Franziska M. Korb, and Tobias Egner \\ Center for Cognitive Neuroscience, Duke University, Durham, North Carolina 27708
}

Cognitive models have long distinguished between "automatic" associative processes that can be triggered in a bottom-up fashion, and "controlled" processes, where internal goals guide information processing in a deliberate, top-down manner. However, recent behavioral studies have cast doubt on the validity of this dichotomy, showing that implicit contextual cues can modulate performance in a way suggestive of an associative triggering of specific top-down control states. Here, we harnessed functional magnetic resonance imaging in humans to test whether these behavioral findings truly reflect online, bottom-up priming of top-down attentional control settings. Using a flanker interference task where stimulus location cued the likelihood of incongruent trials, we found that the behavioral phenomenon of implicit, context-specific improvements in interference resolution was mirrored in hemodynamic activity in the medial superior parietal lobule (mSPL), previously implicated in voluntary (as opposed to primed) attention shifts. Moreover, the mSPL displayed context-specific functional coupling with visual regions involved in processing the flanker stimuli, and the modulation of the latter was predictive of the behavioral effects. Finally, the implementation of this contextual control was "on the fly," that is, it was primed online by a switch to the context associated with high conflict. These results suggest that top-down control states can be bound into episodic event representations and can subsequently be primed by other features of those representations. Together, our findings illustrate a more intimate link between associative and controlled processing than is traditionally assumed, and place the neural substrate of that linkage in the posterior parietal cortex.

\section{Introduction}

Cognitive theories traditionally distinguish between involuntary, "automatic" processing based on well learned stimulus-response (S-R) associations that are triggered in a bottom-up fashion, and effortful "controlled" processing that employs internal goal representations to intentionally overcome habitual associations in a top-down manner (Schneider and Shiffrin, 1977; Norman and Shallice, 1986). Over the years, this view has inspired many competing explanations of cognitive phenomena. For instance, "conflict adaptation," the finding that the frequent or recent experience of conflicting stimulus or response features facilitates resolution of such interference has been cast either as reflecting strategic top-down control processes (Botvinick et al., 2001; Egner and Hirsch, 2005) or bottom-up S-R associations (Mayr et al., 2003; Hommel et al., 2004).

Recent behavioral findings, however, suggest that this juxtaposition of automatic and controlled processing may in fact represent a false dichotomy. Specifically, it has been documented that a nominally irrelevant contextual cue that is paired with

Received Feb. 27, 2012; revised April 19, 2012; accepted April 28, 2012.

Author contributions: J.A.K., F.M.K., and T.E. designed research; J.A.K. performed research; J.A.K. analyzed data; J.A.K., F.M.K., and T.E. wrote the paper.

This research was funded by National Institute of Mental Health Award R01MH087610 (T.E.). We thank Chris Blais, Yu-Chin Chiu, Matthew Crump, and Markus Ullsperger for their helpful comments on a previous version of this manuscript and Jiefeng Jiang for providing custom brain rendering software.

Correspondence should be addressed to Tobias Egner, Center for Cognitive Neuroscience, Duke University, Box 90999, Durham, NC 27708. E-mail: tobias.egner@duke.edu.

DOI:10.1523/JNEUROSCI.0934-12.2012

Copyright $\odot 2012$ the authors $\quad 0270-6474 / 12 / 328192-09 \$ 15.00 / 0$ frequent conflict can elicit context-specific improvements in interference resolution (Corballis and Gratton, 2003; Crump et al., 2006; Lehle and Hübner, 2008; Vietze and Wendt, 2009; Wendt et al., 2008) - even though observers are unaware of the contextual manipulation. Given the context-specificity and implicit nature of this effect, it must be driven by bottom-up stimulus features. At the same time, however, the effect appears immune to a purely associative explanation, because it generalizes to novel stimuli (Crump and Milliken, 2009; Heinemann et al., 2009). Thus, contextual cues do not appear to prime specific S-R associations, but rather the rapid (and implicit) retrieval and implementation of particular top-down control settings.

This has raised the intriguing prospect of "priming of control," the possibility that top-down control may be subject to associative bottom-up retrieval, triggered by stimulus context (Verguts and Notebaert, 2008, 2009; Spapé and Hommel, 2008). However, behavioral data alone are limited in their ability to establish that the phenomenon of context-specific improvements in interference resolution truly reflects control, that is, top-down modulation of stimulus-driven processing. Additionally, such effects may not necessarily reflect contextual priming of transient, online control adjustments upon exposure to the contextual cue, but rather a strategic (albeit implicit) sustained attentional focus to the high-conflict context.

Here, we tested the priming of control hypothesis by pairing functional magnetic resonance imaging (fMRI) with a variant of the Eriksen flanker task (Eriksen and Eriksen, 1974) where the ratio of low- to high-conflict stimuli varied according to visual hemifield (Fig. 1A). This design allowed us, first, to delineate po- 
tential neural sources of context-specific improvements in interference resolution, by identifying brain regions displaying differential patterns of activation associated with conflict processing for stimuli presented in the high- versus low-conflict location; and second, to test whether such contextual effects are implemented by biasing task-related sensory processing in visual cortex. Finally, by exploring variation in the behavioral and neural indices of interference resolution as a function of context transitions (i.e., repetitions vs switches), we could address the question as to whether contextual cues prime control settings "on the fly" or induce a sustained upregulation of processing selectivity.

\section{Materials and Methods}

Participants. Thirty right-handed volunteers gave written informed consent to participate in this study, which was approved by the Duke University Health System Institutional Review Board. All participants had normal or correctedto-normal vision, and reported no current or history of neurological, psychiatric, or major medical disorder. They were reimbursed with $\$ 30$ for their participation, which lasted $\sim 75$ min. The data of five participants were excluded-two for incomplete scans, two for chance level performance, and one for movement-related artifacts. The remaining 25 participants were 14 females and 11 males (mean age $=23.8$ years; range $=18-33$ years).

Procedure and stimuli. Participants performed two tasks in counterbalanced order during fMRI: a face-viewpoint classification version of the Eriksen flanker task including a contextual location-based manipulation of conflict frequency (Fig. 1A) and a localizer task designed to identify face-sensitive regions of extrastriate cortex ["fusiform face area" (FFA); Kanwisher et al. (1997)], and location-sensitive regions of early visual cortex in which we could probe contextual modulation of stimulusdriven sensory processing during the main experiment. Face stimuli for both tasks were generated using FaceGen software (Singular Inversions) to produce an equal number of left- and right-looking male and female faces (137 each; viewpoint angle: $\sim 45-50^{\circ}$ ) with unique identities from various age- and ethnic-groups. More specifically, the program was used to generate a random face from the "All Races" sub-tab, which was then adjusted using both the shape and color controls on the "Gender" and "Age" scales while locking the "Caricature" and "Asymmetry" scales at "average" and "typical," respectively. Only faces with unambiguous gender features were selected under consensus of the first author and a graduate-level research assistant. Additionally, we used the "Race Morphing" scales to create an approximately equal number of faces from each of the four possible categories (African, European, Southeast Asian, East Indian), including faces with mixed racial identity. A total of 548 face images were used, one for each face trial of the localizer task and each trial of the main flanker experiment. Localizer stimuli consisted of an additional 100 house images selected from local real estate websites and 100 Fourier-scrambled face and house images (50 each). Stimuli were presented against a black background on a back-projection screen, which participants viewed in a mirror mounted to the head coil. This setup simulated a viewing distance of $\sim 80 \mathrm{~cm}$, resulting in individual stimuli extending $\sim 0.72^{\circ}$ horizontally and $1.1^{\circ}$ vertically. Participants responded with right-hand index and middle finger button presses on an MRI-compatible response box (Current Designs), which was vertically oriented on the participant's chest (in plane with the length of their body) to counteract potential spatial S-R compatibility effects during performance of the flanker experiment. Task programming, stimulus presentation, and behavioral recording were performed with Presentation software (Neurobehavioral Systems).

Tasks. During the localizer scan, participants performed a one-back task on streams of face, house, or scrambled images (between pseudorandomly interspersed fixation blocks), which were presented at one of 10 positions corresponding to the coordinates of individual faces in flanker stimulus arrays $\left(\sim 2.9^{\circ}, 3.8^{\circ}, 4.7^{\circ}, 5.6^{\circ}, 6.5^{\circ}\right.$ horizontal visual angle to the left and right of central fixation). There were 10 pseudorandomly presented epochs of each stimulus type (houses, faces, and scrambled images), each including 10 stimulus presentations $(750 \mathrm{~ms}$ ) with a fixed interstimulus interval of $250 \mathrm{~ms}$.

In each of the 448 trials of the main flanker experiment (Fig. $1 A$ ), a stimulus array (row of five identical trial-unique face images) was presented pseudorandomly either to the left or right of fixation. Participants were instructed to rapidly and accurately classify the viewpoint direction of the face in the center of the array (target) with a button press (S-R mapping was counterbalanced across subjects) and ignore the flanker faces (distracters). The target face was presented for $320 \mathrm{~ms}$; its onset was delayed by $80 \mathrm{~ms}$ from the onset of the flanker faces, which were shown for $400 \mathrm{~ms}$. Target and flanker face viewpoint direction was congruent in half of all trials and incongruent in the other. Conflict frequency was manipulated according to stimulus location by defining one side of fixation as a high-conflict context ( $25 \%$ congruent/75\% incongruent trials) and the other as a low-conflict context (75\% congruent/25\% incongruent trials; counterbalanced across participants). The task was broken down into four runs of 112 trials each. Interstimulus intervals were jittered between 3 and $5 \mathrm{~s}$ as randomly drawn from a pseudo-exponential distribution, where $50 \%$ of intervals lasted 3 s, $25 \%$ lasted 3.5 s, $12 \%$ lasted $4 \mathrm{~s}, 6 \%$ lasted $4.5 \mathrm{~s}$ and $6 \%$ lasted $5 \mathrm{~s}$, resulting in a mean interval of $\sim 3.5 \mathrm{~s}$. 
Behavioral data analysis. Before analyzing the performance data, we excluded the first trial of each run and all trials with excessively fast/slow responses $(>150 \mathrm{~ms} /<2000 \mathrm{~ms} ; 1.6 \%$ of all trials). Our primary analyses tested for contextual variation in interference effects (incongruent congruent) in mean reaction times (RT) on correct trials (excluding posterror trials) and error rates with 2 (context: high-conflict vs lowconflict) $\times 2$ (congruency: congruent vs incongruent) repeatedmeasures ANOVAs. Control analyses explored whether contextual variation in interference effects (context $X$ congruency interactions) was mediated by cumulative trial-by-trial effects of conflict [i.e., conflict adaptation; cf. Egner (2007)] with 2 (trial $n-1$ congruency) $\times 2$ (trial $n$ context $) \times 2$ (trial $n$ congruency) ANOVAs. Finally, to obtain evidence whether the phenomenon of context-specific improvements in interference resolution reflects transient, online retrieval of control settings upon exposure to the contextual cue or rather a sustained attentional bias to the high-conflict context, we reanalyzed the RT data with 2 (context transition: repetition vs switch) $\times 2$ (context) $\times 2$ (congruency) ANOVAs.

Post-test questionnaire. Immediately following the scanning session, we used a survey designed to quantify participants' awareness of contextual variation in conflict frequency in the flanker task. First we showed example illustrations of low-conflict congruent and high-conflict incongruent stimulus arrays and asked "Did you notice any systematic variation in how often the direction in which target faces and distracter faces were looking was the same direction (i.e., were congruent) or different direction (i.e., were incongruent)?" The survey then informed participants that conflict frequency may have been manipulated according to face gender (female vs male), skin color (dark vs light), or stimulus location (left vs right of central fixation) and asked them to rate the frequency at which incongruent arrays occurred for each possibility on six corresponding visual analog scales $(0 \%=$ never, $50 \%=$ balanced, $100 \%=$ always). Finally, participants were verbally informed that $75 \%$ of all trials presented on one side of fixation were congruent and $75 \%$ of the trials on the other side of fixation were incongruent and then asked to make a forced choice decision as to which side of the monitor they thought had a greater proportion of incongruent trials.

Image acquisition. Imaging was conducted on a GE Discovery MR750 system at 3.0 tesla using a standard head coil. During both tasks, we acquired functional images parallel to the AC-PC plane with a $\mathrm{T}^{*}$ weighted single-shot gradient EPI sequence of 36 contiguous axial slices [repetition time, $2000 \mathrm{~ms}$; echo time, $28 \mathrm{~ms}$; flip angle, $90^{\circ}$; field of view, $192 \mathrm{~mm}$; array size, $64 \times 64$ ] with $3 \mathrm{~mm}$ thickness and $3 \times 3 \mathrm{~mm}$ in-plane resolution. Structural images were acquired with a T1-weighted FSPGR axial scan using a 3D inversion recovery prepared sequence, recording 120 slices of $1 \mathrm{~mm}$ thickness and in-plane resolution of $1 \times 1 \mathrm{~mm}$. All preprocessing and statistical analysis was performed using SPM8 (http:// www.fil.ion.ucl.ac.uk/spm/software/spm8/). Functional data were slicetime corrected and spatially realigned to the first volume of the respective task. The realigned images were normalized to the MNI template brain (resampled voxel size $2 \mathrm{~mm}^{3}$ ). The first 10 volumes of each run in which no stimulation occurred were discarded before estimating statistical models. A $128 \mathrm{~s}$ temporal high-pass filter was applied to the data and models. Temporal autocorrelations were estimated using restricted maximum likelihood estimates of variance components with a first-order autoregressive model, and the resulting nonsphericity was used to form maximum likelihood estimates of activation.

Image analysis. For the localizer task data, epochs of face, house, and scrambled stimuli presented left and right of fixation and null blocks were each modeled with separate box-car functions convolved with a canonical hemodynamic response function (HRF). Statistical parametric maps (SPMs) of cortical sensitivity to face stimuli (face $>$ house + scrambled images) and stimulus location (left $>$ right stimulation side and right $>$ left stimulation) were calculated for each subject and entered into voxelwise one-sample $t$ tests, where participants were treated as random-effects. Location- and face-sensitive ROIs were defined as voxels with $Z$-standardized $t$ values $>2.58(p<0.005)$ belonging to clusters ( $\geq 24$ contiguous voxels) in the resulting group average maps located within the small volumes of the bilateral combined calcarine sulcus/ lingual gyrus (early visual cortex; left hemisphere, 4003 voxels; right hemisphere, 3907 voxels) and fusiform gyrus (FFA; 3508 voxels) [as defined with the WFU PickAtlas (http://fmri.wfubmc.edu/software/ PickAtlas/)], corresponding to a cluster extent-corrected threshold of $p<0.05$ [as determined by AFNI's 3dClustSim (http://afni.nimh.nih. gov/pub/dist/doc/program_help/3dClustSim.html) according to the procedure described in detail for the main experiment below]. Due to a high degree of variability in the spatial extent of activations between cortical regions and individuals for a given analysis at a specific statistical threshold, we constructed $5 \mathrm{~mm}$ spherical ROIs centered on peak activations in each region for each participant for further analysis of data acquired during the flanker experiment.

For the flanker task data, event-related regressors convolved with a canonical HRF were created corresponding to the stimulus onsets of congruent and incongruent trials in the low- and high-conflict contexts. Error trials, posterror trials, trials with excessively slow/fast responses (12.5\% of all trials), and the first trial of each run were modeled separately as nuisance variables. Single-subjects contrasts were calculated to establish the hemodynamic correlates of conflict processing (incongruent $>$ congruent), context representation (highconflict context $>$ low-conflict context), and, most importantly, contextual variation in conflict processing (context $\times$ congruency interaction, interference $_{\text {high-conflict context }}>$ interference low-conflict context $)$. Group effects were assessed by submitting the individual SPMs to voxelwise one-sample $t$ tests (random-effects model). To identify brain regions in which the hemodynamic expression of context-specific improvements in interference resolution correlated with the behavioral expression of this effect, we entered each participant's context $\times$ congruency interaction SPM into a secondlevel random effect group regression analysis, together with their individual behavioral context-specific interference effect scores (calculated from RTs as interference $_{\text {low-conflict context }}-$ interference high-conflict context $_{\text {f }}$ and, to control for between-subject variability in overall response speed, individual mean $\mathrm{RT}$ as covariates.

To control for false-positive rates, combined voxel activation intensity and cluster extent thresholds corrected for multiple comparisons were determined by using $3 \mathrm{dClustSim}$. Specifically, the program was used to run 10,000 Monte Carlo simulations taking into account the whole-brain search volume and the estimated smoothness of each axis of the respective group SPMs to generate probability estimates of a random field of noise producing a cluster of voxels of a given extent for a set of voxels passing a specific voxelwise $p$ value threshold, which we set at 0.005 for all analyses. Given this voxelwise threshold, the simulations determined that cluster sizes $>118-190$ voxels, depending on the specific analysis, corresponded to combined threshold of $p<0.05$ (corrected).

Psychophysiological interaction analysis. Psychophysiological interaction (PPI) analysis provides a measure of functional connectivity by quantifying covariation of activation time courses between different brain regions (the "physiological" variable) depending on a specific experimental condition (the "psychological" variable) (Friston et al., 1997). We conducted a PPI analysis to assess the functional connectivity between a region of the right medial superior parietal cortex (mSPL) identified by the context $\times$ congruency interaction contrast and the behaviorally informed regression analyses described above (compare Fig. 3 ), and potential target regions of top-down modulation in sensory cortex (independently localized early visual cortex and FFA ROIs) as a function of context-specific variation in conflict processing. To this end, we first extracted the deconvolved activation time course from a $5 \mathrm{~mm}$ radius sphere centered on the peak mSPL activation and then calculated the product of this activation and the vector of the psychological variable (context $\times$ congruency interaction, interference high-conflict context $>$ interference $\left._{\text {low-conflict context }}\right)$ to create the PPI term. New models were generated including as regressors the PPI term, the physiological variable, and the psychological variable. For each participant, we tested whether activity within the early visual cortex and/or FFA ROI volumes could be predicted by the PPI term, with the physiological and psychological regressors treated as nuisance variables. Finally, the resulting individual PPI SPMs were entered into a voxelwise group one-sample $t$ test, and activation within the combined early visual cortex and FFA ROIs (small volume: 453 voxels) was assessed using $3 \mathrm{dClustSim}$ as described above. Given this volume, simulations determined that minimum cluster 
extent $>13$ voxels corresponded to a corrected $p<0.05$ threshold. To probe the process-specificity of the results, we conducted two additional PPI analyses based on blood oxygenation level-dependent (BOLD) activity recorded in the $\mathrm{mSPL}$ using the main effect of conflict processing (incongruent $>$ congruent) and context representation (high-conflict context $>$ low-conflict context) as psychological variables.

ROI analyses. For evaluation of activity in empirically defined ROIs, we extracted mean $\beta$ estimates using Marsbar software (http://marsbar. sourceforge.net/) for each participant on conditions of interest from a 5 $\mathrm{mm}$ radius sphere centered on activation maxima and submitted the resulting values to statistical tests. Given the lateralization of BOLD responses in visual cortex for laterally presented stimuli (contralateral $>$ ipsilateral) and that our spatial contextual manipulation was counterbalanced across participants, we investigated contextual modulation in the early visual cortex and FFA ROIs by submitting the individual $\beta$ estimates to 2 (laterality of ROI: ipsilateral vs contralateral cortical hemisphere relative to the high-conflict hemifield) $\times 2$ (context) $\times 2$ (congruency) ANOVAs, rather than analyzing the data separately for left and right anatomical ROIs.

\section{Results}

\section{Behavioral data and post-test questionnaire results}

Overall performance of the flanker task was high ( $93 \%$ correct) and characterized by typical interference effects. RTs were slower for incongruent stimuli (783 ms) than for congruent arrays (674 ms; $\left.F_{(1,24)}=133.8 ; p<0.0001\right)$. Similarly, error rates were elevated on incongruent $(9.8 \%)$ relative to congruent trials $(3.8 \%$; $\left.F_{(1,24)}=20.6 ; p<0.0001\right)$. More importantly, although RTs did not vary generally as a function of the contextual conflict frequency manipulation (high-conflict context: $727 \mathrm{~ms}$ vs lowconflict context: $729 \mathrm{~ms} ; F_{(1,24)}=0.15$; n.s.), interference effects were reduced for stimuli presented in the high-conflict location (94 ms) relative to those in the low-conflict context $(123 \mathrm{~ms}$; $F_{(1,24)}=7.6 ; p=0.01 ;$ Fig. $\left.1 B\right)$. This context-specific variation in the efficiency of interference resolution could not be attributed to a speed-accuracy tradeoff, as the accuracy data reflected a qualitatively identical pattern with no general variation in error rates as a function of conflict frequency (high conflict: $6.5 \%$ vs low conflict: $7.1 \% ; F_{(1,24)}=0.9$; n.s.) and a trend indicative of reduced interference in the high-conflict context $(5.1 \%)$ relative to low-conflict context $\left(7.0 \% ; F_{(1,24)}=2.7 ; p=0.1\right.$; Fig. $\left.1 C\right)$.

Control analyses documented that reduced interference in the high-conflict context was not qualified by trial-by-trial effects of stimulus congruency [i.e., conflict adaptation; cf. Egner (2007)], corroborating previous reports (Crump et al., 2006). Specifically, although conflict adaptation (i.e., trial $n-1$ congruency $\times$ trial $n$ congruency interactions indicating reduced interference effects immediately following incongruent trials) was evident both in RTs and error rates (both $F_{(1,24)}>5.9$; both $p<0.05$ ), contextual variation in interference effects (i.e., context $\times$ congruency interactions; RTs: $F_{(1,24)}>8.0 ; p<0.01$; error rates: $F_{(1,24)}=$ $2.5 ; p=0.12)$ was not influenced by congruency sequences (both $F_{(1,24)}<0.2 ;$ n.s.).

The proposal that context-specific improvements in interference resolution reflect implicit priming of control is supported by findings suggesting that the behavioral phenomenon occurs in the absence of awareness regarding contextual variation of conflict frequency (Crump et al., 2006; Heinemann et al., 2009; Sarmiento et al., 2012). In line with these previous results, only one of 25 participants in the current experiment reported noticing the location-based manipulation of conflict frequency in our posttest survey (see Materials and Methods). Further, participants' ratings on the respective visual analog scales pertaining to the spatial conflict frequency manipulation indicated that they perceived the ratio of incongruent to congruent stimuli to be evenly balanced between the two sides of fixation $(51.1, \mathrm{SD}=4.6)$, and these ratings did not diverge either from those on the scales gauging attributions of conflict frequency to face gender $(49.1, \mathrm{SD}=$ $4.6)$ or skin color $\left(50.7, \mathrm{SD}=4.6\right.$; one-way $\operatorname{ANOVA~} F_{(1,24)}=1.4$; n.s.). Interestingly, however, after we informed the participants that conflict frequency was manipulated according to stimulus location and not by face gender or skin color, 17 individuals correctly reported which side of the display had a greater proportion of incongruent trials in their version of the experiment, while only 8 answered incorrectly (binomial test; $p=0.054$ ), suggesting that they might have had some implicit knowledge of the actual contextual manipulation.

In sum, the behavioral data successfully replicated the phenomenon of context-specific improvements in interference resolution, and the post-test questionnaire confirmed that it occurs implicitly, thus setting the stage for determining the underlying neural mechanism.

\section{fMRI data: conflict processing, context representation, and context-specific variation in conflict processing}

An initial random-effects analysis of the fMRI data confirmed an expected pattern of conflict-related activation (incongruent $>$ congruent) in posterior medial frontal cortex including the anterior cingulate and presupplementary motor area, lateral prefrontal cortex, and inferior parietal lobule (Fig. 2A, Table 1), consistent with previous studies of conflict processing (Ridderinkhof et al., 2004; Nee et al., 2007). Although stimulus context had no general influence on behavioral performance and participants were unaware of the contextual conflict frequency manipulation, an exploratory analysis of the main effect of context in the fMRI data (high-conflict context $>$ low-conflict context) uncovered a region of the right lateral superior parietal lobule showing generally elevated activation for stimuli presented in the high-conflict context (Fig. 2 B, Table 1).

Having identified brain regions generally involved in conflict processing and context representation in our task, we addressed the primary objective of elucidating regions mediating the integration of information regarding contextual variation in conflict frequency and the degree of interference on the current trial. To this end, we calculated the critical context $\times$ congruency interaction (interference high-conflict context $>$ interference $_{\text {low-conflict context }}$ ). Two regions emerged displaying differential patterns of conflict processing for stimuli presented in the high- versus low-conflict context: the left dorsal premotor cortex and the right medial superior parietal lobule (Fig. $3 A$, Table 1). At a slightly more lenient extent threshold, mSPL activation of comparable intensity was also evident in the left hemisphere $(x-12, y-58, z 62$; $T_{\max }=4.16 ; 71$ voxels). It should be noted, however, that the precise pattern of the context $\times$ congruency interactions evident in these regions (all $F_{(1,24)}>10.6$; all $p<0.005$; Fig. $3 B$ ) was not conforming to the expectation that superior conflict resolution in the high-conflict context would be expressed as increased activity on incongruent versus congruent trials. Specifically, although activation was relatively greater in response to incongruent trials in the high- versus low-conflict context in these regions (all $t_{(24)}>$ 2.7 ; all $p<0.05$ ), there was no contextual modulation of activity on congruent trials (all $t_{(24)}<0.98$; all $p>0.1$ ) and this stable response was equally strong as activity on incongruent trials in the high-conflict context (all $t_{(24)}<1.5$; all $p>0.1$ ). Thus, the differential pattern of conflict processing in the high- versus lowconflict contexts in these regions was primarily attributable to the relatively low response to incongruent versus congruent trials in the low-conflict context (all $t_{(24)}>1.9$; all $p<0.07$ ). 
To determine whether this pattern of activation was related to the behavioral improvement in interference resolution in the high-conflict context, we conducted a complementary behaviorally informed regression analysis of the context $\times$ congruency interaction in the fMRI data, exposing regions in which the hemodynamic expression of context-specific variation in conflict processing correlated with the behavioral expression of this effect across participants (see Materials and Methods). Significant brain-behavior relationships were revealed in the bilateral temporoparietal junction, cerebellum, and, most importantly, the same region of the right $\mathrm{mSPL}$ identified by the conventional context $X$ congruency interaction analysis as described above (Fig. 3C,D; Table 1). At a slightly more lenient extent threshold, correlations between the hemodynamic and behavioral expression of contextual variation in interference resolution were also evident in the left mSPL $\left(x-16, y-56, z 72 ; T_{\max }=4.26 ; 153\right.$ voxels; Fig. 3D).

\section{Context-specific improvements in interference resolution reflect top-down control}

The contextual variation in conflict processing and associated brain-behavior relationships revealed in mSPL activity suggest that this region is centrally involved in mediating interference reduction in the high- versus low-conflict context, but do not establish that this role is one of executing top-down control over task-related sensory processing. If this were the case, one would expect the mSPL to display greater functional connectivity with potential target regions of control in visual cortex during conflict processing in the high- versus low-conflict context. We therefore conducted a PPI analysis (Friston et al., 1997) to assess coupling between this region and the independently localized early visual cortex and FFA ROIs as a function of contextual variation in conflict processing (interference ${ }_{\text {high-conflict context }}>$ interference $_{\text {low-conflict context }}$; see Materials and Methods).

In line with a role of the mSPL in contextual conflict-control, BOLD activity in this region associated with differential conflict processing in the high- versus low-conflict context significantly covaried with that in both visual cortex ROIs (Fig. $4 A$ ). We questioned whether this pattern of connectivity was specific to the process of contextually cued adjustments in conflict processing or might merely reflect a generic response to the demands of our task. To address this possibility, we conducted two control PPI analyses, testing whether similar patterns of connectivity between the MSPL and visual cortex were evident as a function of general conflict processing (incongruent $>$ congruent) or context representation (high conflict $>$ low conflict). However, neither control analysis revealed significant covariation with any posterior regions, including either the early visual cortex or FFA ROIs (Fig. $4 B$ ).

Contextually cued adjustments in top-down control settings might facilitate context-specific improvements in interference resolution in the current task by amplifying target processing, suppressing distracter processing, biasing spatial attention, or some combination of these possibilities. To explore the manner in which contextual conflict-control modulated stimulus processing, we submitted BOLD activity recorded from the early visual cortex and FFA ROIs to 2 (hemispheric laterality of ROI: ipsilateral versus contralateral relative to the high-conflict hemifield) $\times 2$ (context: low- conflict vs high-conflict) $\times 2$ (congruency: congruent vs incongruent) repeated-measures ANOVAs (see Materials and Methods). In early visual cortex, an expected laterality $\times$ context interaction reflecting spatially driven lateralization (contralateral $>$ ipsilateral) was evident $\left(F_{(1,24)}=37.8 ; p<0.0001\right)$, but no modulation suggestive of top-down biasing was detected (Fig. $4 C$ ). In contrast, a laterality $\times$ context interaction in the FFA $\left(F_{(1,24)}=8.0 ; p<\right.$ 0.01 ) was driven by a pattern indicative of context-specific control over spatial attention. Specifically, while lateralization was present in the FFA for stimuli presented in the low-conflict context $\left(t_{(24)}=3.0\right.$; $p<0.01$ ), it was absent for stimuli presented in the high-conflict context (i.e., both hemispheres were equally recruited by stimuli presented in the high-conflict hemifield; $t_{(24)}=1.2$; n.s.; Fig. $4 C$ ). Reduced stimulus-driven lateralization in sensory cortex is concordant with recent reports of spatially global gain modulation under conditions of elevated cognitive control (Ester et al., 2009; King et al., 2010). Accordingly, the magnitude of contextual lateralization re-

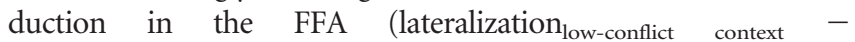

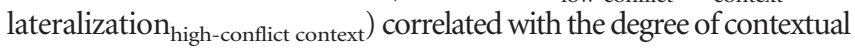
interference reduction in behavior (interference ${ }_{\text {low-conflict context }}-$ interference $_{\text {high-conflict context: }}: r=0.51 ; p<0.01$ ) across participants.

\section{Contextual conflict-control is primed online}

The findings thus far support the hypothesis that context-specific improvements in interference resolution effects reflect implicit top-down adjustments in processing selectivity (Crump et al., 2006; Lehle and Hübner, 2008; Wendt et al., 2008), but are not directly informative to the question as to whether contextual cues prime retrieval of control settings rapidly on the fly upon stimulus presentation or induce a sustained attentional focus on the high-conflict context that is maintained across trials. If contextual cues primed a sustained increase of attention on the highconflict context, then superior interference resolution should be evident immediately upon a transition from the low- to highconflict context, because heightened attentional focus would already be present for that location. By contrast, if attentional control were retrieved on the fly in an online fashion whenever stimuli appeared in the high-conflict context, then it could be expected that reduced interference effects would not be immediately evident upon a transition from the low- to high-conflict context, because control settings would have to be retrieved first. To adjudicate between these possibilities, we first reanalyzed the RT data, testing whether improvements in interference resolution were immediately present following a switch from the low- to high-conflict context, or were evident only following a repetition of the high-conflict context (context transition $\times$ context $\times$ congruency ANOVA). The three-way interaction was significant $\left(F_{(1,24)}=10.9 ; p<0.005\right)$, such that reduced interference 
Table 1. Activations revealed by the conflict, context, context $\times$ congruency interaction, and behaviorally informed context $\times$ conflict interaction analyses

\begin{tabular}{|c|c|c|c|c|c|c|}
\hline Anatomical area & Hemisphere & $x$ & $y$ & $z$ & Extent & $T_{\max }$ \\
\hline \multicolumn{7}{|l|}{ Conflict (incongruent > congruent) } \\
\hline Precentral gyrus & $\mathrm{L}$ & -30 & -12 & 58 & 218 & 4.04 \\
\hline Posterior medial frontal cortex & $\mathrm{L} / \mathrm{R}$ & -6 & 0 & 56 & 745 & 5.34 \\
\hline Inferior parietal lobule & L & -40 & -46 & 48 & 1067 & 4.68 \\
\hline Inferior parietal lobule & $\mathrm{R}$ & 42 & -48 & 44 & 2403 & 6.75 \\
\hline Precuneus & $\mathrm{L}$ & -26 & -66 & 38 & 126 & 3.96 \\
\hline Inferior frontal gyrus & $\mathrm{R}$ & 52 & 4 & 36 & 844 & 5.55 \\
\hline Inferior frontal gyrus & $\mathrm{L}$ & -36 & 24 & 22 & 184 & 3.93 \\
\hline Anterior insula/frontal operculum & $\mathrm{R}$ & 42 & 16 & -10 & 191 & 3.83 \\
\hline Inferior temporal gyrus & $\mathrm{R}$ & 54 & -56 & -16 & 134 & 3.85 \\
\hline \multicolumn{7}{|l|}{ Context (high conflict $>$ low conflict) } \\
\hline Lateral superior parietal lobule & $\mathrm{R}$ & 24 & -54 & 66 & 345 & 3.52 \\
\hline \multicolumn{7}{|c|}{ Context $\times$ congruency interaction (interference high conflict $>$ interference $_{\text {low conflict }}$ ) } \\
\hline Superior frontal gyrus & $\mathrm{L}$ & -22 & 12 & 68 & 239 & -3.71 \\
\hline Medial superior parietal lobule & $\mathrm{R}$ & 14 & -46 & 66 & 427 & 4.37 \\
\hline \multicolumn{7}{|c|}{ Behaviorally-informed context $\times$ congruency interaction } \\
\hline Medial superior parietal lobule & R & 8 & -50 & 66 & 451 & 3.84 \\
\hline Temporoparietal junction & $\mathrm{L}$ & -60 & -40 & 20 & 775 & 4.75 \\
\hline Temporoparietal junction & $\mathrm{R}$ & 66 & -34 & 20 & 407 & 4.33 \\
\hline Anterior cerebellum/lingual gyrus & $\mathrm{L}$ & -24 & -48 & -26 & 335 & 3.92 \\
\hline
\end{tabular}

R, right; L, left.

\section{A}

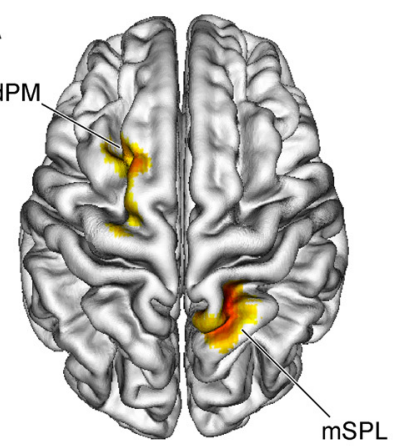

C

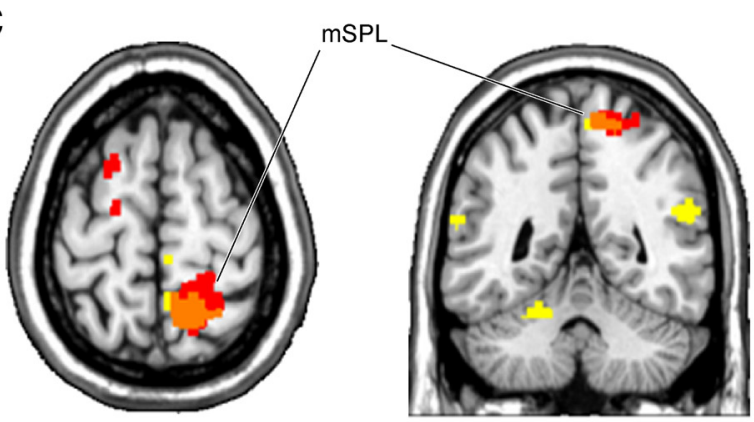

B

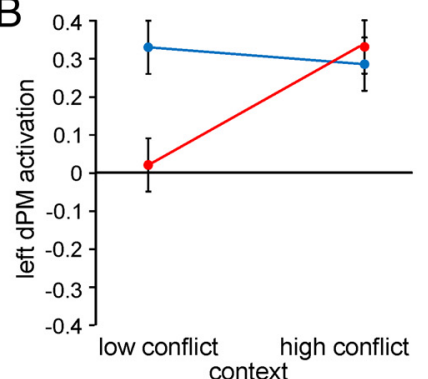

context

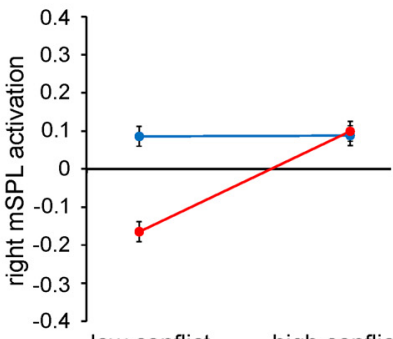

context

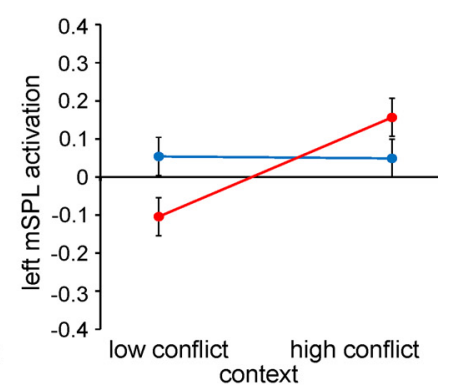

context
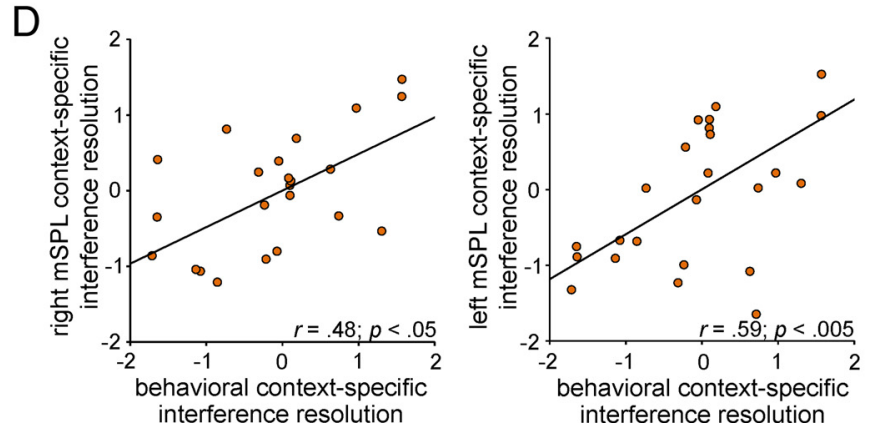

Figure 3. Neural substrates of context-specific variation in conflict processing. $A$, Group-averaged $T$-map ( $p<0.05$, corrected) depicting activation associated with context-specific variation in conflict processing (interference high-conflict context $>$ interference Iow-conflict context $_{\text {) }}$ is plotted on a 3-D rendering of an individual brain normalized to the MNI template. dPM, dorsal premotor cortex. mSPL activation of comparable intensity was evident in the left hemisphere at an uncorrected extent threshold $\left(x-12, y-58, z 62 ; T_{\max }=4.16 ; 71\right.$ voxels; data not shown). $\boldsymbol{B}$, Group mean activation in the left $\mathrm{dPM}$, right $\mathrm{mSPL}$, and left $\mathrm{mSPL}$ ( $\beta$ estimates \pm SEM) is plotted for congruent and incongruent trials as a function of conflict frequency context, illustrating the critical interactions in each region: all $F_{(1,24)}>10.6$; all $p<0.005$. C, Regions identified by the behaviorally informed context $\times$ congruency regression analysis are plotted (yellow) together with the results of the conventional interaction analysis (red) at $p<0.05$ (corrected) on axial ( 264$)$ and coronal ( $y-48$ ) slices of an individual brain. The orange region represents the conjunction of the two effects. Brain- behavior relationships were also evident in the left mSPL at a slightly more lenient extent threshold $\left(x-16, y-56, z 72 ; T_{\max }=4.26 ; 153\right.$ voxels). $\boldsymbol{D}$, Correlation between individual mean levels of BOLD activity (z-normalized $\beta$ estimates) in the right and left mSPL associated with contextual variation in conflict processing (calculated as the difference between in interference effects in the high-conflict versus low-conflict contexts, i.e., interference ${ }_{\text {high-conflict context }}-$ interference $_{\text {low-conflict context }}$ ) and the individual $z$-normalized behavioral context-specific interference

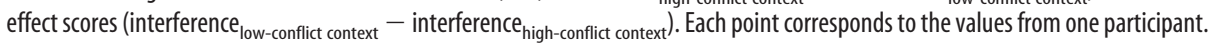

effects were not evident following a transition from the low- to highconflict context $\left(t_{(24)}=0.4 ;\right.$ n.s.), but were clearly present on trials where the high-conflict context was repeated $\left(t_{(24)}=4.6 ; p<0.0001\right.$; Fig. 5A). Together, this pattern contradicts the notion that contextual cues induce sustained adjustments in attention and suggests that conflict-control settings are retrieved in an online manner whenever stimulus presentation switches between contexts and then facilitate performance on subsequent trials in that context.

These behavioral findings impose a new constraint on the activity profile of a candidate brain region mediating contextual 
A

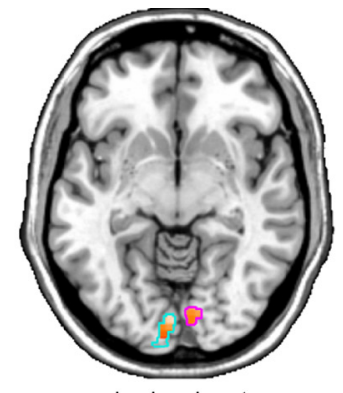

early visual cortex
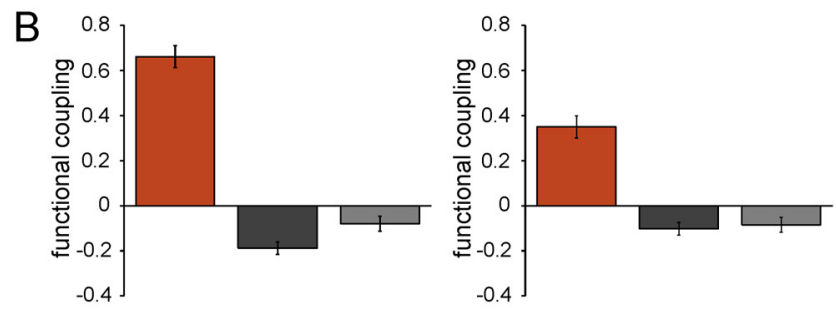

$\square$ conflict $_{\text {high }}>$ conflict $_{\text {low }} \square$ context: high > low $\square$ conflict: incongruent > congruent

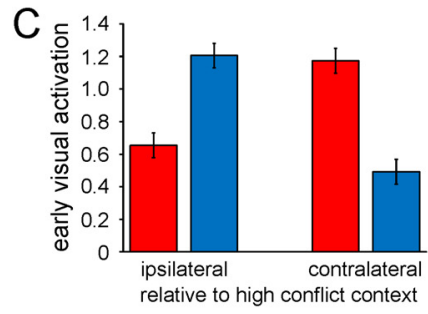

$\square$ high conflict context

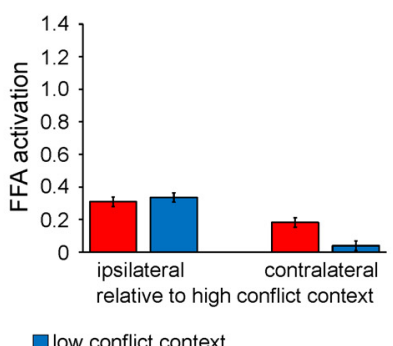

$\square$ low conflict context

Figure 4. Context-specific improvements in interference resolution are mediated by topdown control. $\boldsymbol{A}$, Functional coupling with the right $\mathrm{MSPL}$ as a function of context-specific variation in conflict processing (interference high-conflict context $>$ interference $_{\text {low-conflict context }}$ ) as revealed by PPI analysis is shown for voxels in the independently localized early visual cortex [left panel; left hemisphere (light blue outlines): $x-2, y-86, z-12 ; 290$ voxels; right hemisphere (magenta outlines): $x 10, y-80, z-8 ; 75$ voxels] and FFA ROIs (right panel; green outlines; left hemisphere: $x-44, y-46, z-26 ; 35$ voxels; right hemisphere: $x 48, y-56, z$ $-22 ; 53$ voxels), displayed at $p<0.05$ (small volume correction) on axial $(z-7)$ and coronal $(y-50)$ slices of an individual brain in MNI space. $\boldsymbol{B}$, Greater functional coupling ( $\beta$ estimates \pm SEM) between the mSPL and bilateral early visual cortex (left panel) and FFA (right panel) as a function of context-specific variation in conflict processing (orange) relative to that as a function general context representation (dark gray) and conflict processing (light gray) as revealed by PPI analysis (one-way ANOVAs: both $F_{(1,24)}>29.1$, both $p<0.0001$ ). C, Mean activation ( $\beta$ estimates \pm SEM) in the early visual cortex (left panel) and FFA (right panel) ROls is plotted as a function of the laterality of the anatomical hemisphere (relative to the highconflict location/visual hemifield: ipsilateral vs contralateral) and context (high conflict vs low conflict). Note that activation is not plotted relative to stimulus presentation per se given the counterbalancing of the contextual conflict frequency manipulation across participants.

conflict-control. Specifically, one would expect that region to display contextual variation in conflict processing immediately upon transitions between contexts, when contextual conflictcontrol settings are being primed and retrieved. Consequently, we conducted an equivalent ANOVA on the fMRI data recorded from the identified region of the right mSPL. As anticipated, context-specific variation in conflict processing was evident only upon switches between contexts, but not for context repetitions $\left(F_{(1,24)}=4.5 ; p<0.05\right.$; Fig. $\left.5 B\right)$. In sum, the data suggest that contextual cues prime the retrieval and execution of contextually appropriate conflict-control settings by the mSPL, whose behavioral expression can be observed in context-specific variation in the efficiency of interference resolution on the subsequent trial(s) in that context. These results support the proposal that contex-

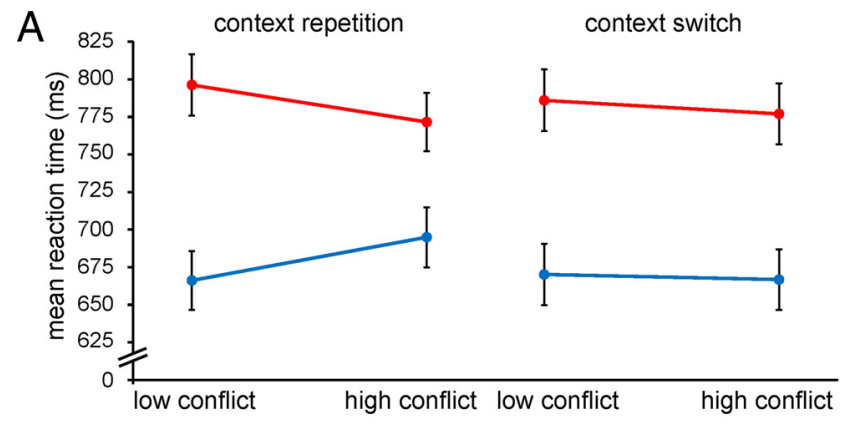

B

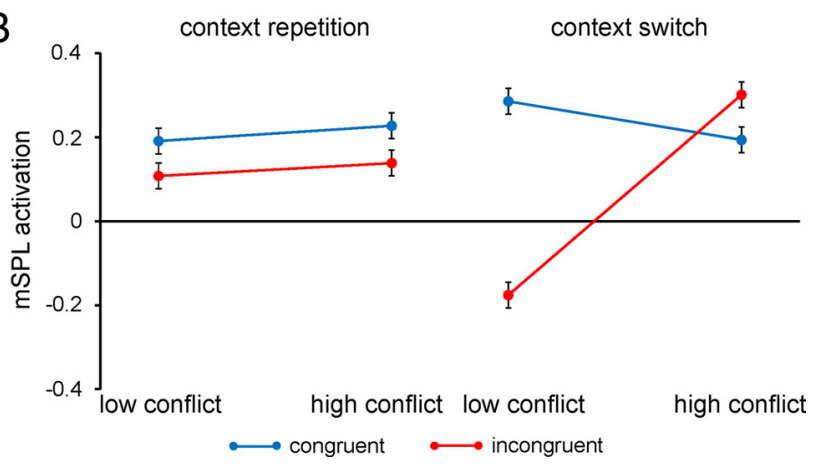

Figure 5. Conflict-control settings are primed by contextual cues. $\boldsymbol{A}$, Group mean RTs ( \pm SEM) are plotted for congruent and incongruent trials as a function of conflict frequency context, separately for context repetitions and context switches. $\boldsymbol{B}$, Group mean right mSPL activation ( $\beta$ estimates \pm SEM) is plotted as in $\boldsymbol{A}$.

tual cues can prime top-down control settings and place the neural substrate of this process in superior parietal cortex.

\section{Discussion}

We used fMRI to test the priming of control hypothesis, the proposition that implicit contextual cues can trigger bottom-up retrieval of specific top-down control settings. First, we showed that the behavioral phenomenon of context-specific improvements in interference resolution was mirrored in the neural expression of context-specific variation in conflict processing in the superior parietal cortex, specifically, the mSPL. Second, mSPL activation was accompanied by a tight coupling with taskrelevant regions in visual cortex, suggesting that contextual conflict-control was mediated by top-down biasing of sensory processing. Finally, we obtained evidence indicating that the implementation of this control state was on the fly, that is, it was primed in a rapid, online manner upon a switch from the low- to high-conflict context, rather than reflecting sustained attentional focus on the high-conflict location across trials.

While many of the behavioral findings that have motivated the priming of control hypothesis (Corballis and Gratton, 2003; Crump et al., 2006, 2008; Lehle and Hübner, 2008; Wendt et al., 2008; Vietze and Wendt, 2009; Wendt and Kiesel, 2011) could alternatively be attributed to simple S-R learning processes (Logan, 1988; Hommel, 2004), recent studies have shown such context-specific improvements in interference resolution to generalize to novel stimuli (Crump and Milliken, 2009; Heinemann et al., 2009). Building on these studies, the current results were obtained in a task using trial-unique stimuli and in which neither stimulus congruency nor conflict frequency context were predictive of a specific response, thus providing conclusive evidence that contextual variation in conflict processing cannot be reduced to S-R associations. Furthermore, contrary to the suspicion that that this phenomenon merely reflects aggregate 
congruency sequence effects, context-specific improvements in interference resolution were dissociable from such trial-by-trial "conflict adaptation" effects in the current study, verifying previous reports (Crump et al., 2006). Crucially, this "pure" expression of contextual conflict-control occurred despite the participant's lack of awareness of systematic variation in conflict frequency, illustrating the implicit nature of the phenomenon and delivering further evidence questioning the notion that controlled processing is inherently volitional (Boy et al., 2010; Schmidt et al., 2007; van Gaal et al., 2010; Blais et al., 2012).

In addition to supporting the basic existence of priming of control, our data shed first light on its underlying neural mechanisms. Specifically, we identified the mSPL to be instrumental in integrating implicit contextual conflict frequency information with the level of conflict on the current trial, and in mediating behavioral improvements in interference resolution in the high-conflict context. These findings extend the established role of the mSPL in mediating explicitly cued shifts of attention (Vandenberghe et al., 2001; Yantis et al., 2002; Serences et al., 2004; Shulman et al., 2009) to include the retrieval and execution of implicitly primed control settings. The mSPL displayed a somewhat unexpected activation pattern in this respect, however, as its response was more one of a failure to engage and properly process infrequent incongruence in the low-conflict context than one of increased engagement to resolve frequent interference in the high-conflict context. The mSPL is known to exhibit decreased activity when attention is held (e.g., on a spatial location, feature, object, or sensory modality) relative to when it is shifted (for review, see Serences and Yantis, 2006; Yantis, 2008). In keeping with these findings, one possible explanation of the relative activation decrease in the mSPL on incongruent trials in the low-conflict context is that when adjustments in attentional control are not explicitly cued (for review, see Serences and Yantis, 2006; Yantis, 2008 ) or implicitly primed (here), this region disengages. In a similar vein, Kelley and Lavie (2011) recently reported a negative interference effect in the mSPL (such as that observed here in the low-conflict context) under conditions of low working memory load. Future studies could potentially delineate the functional characteristics of this region more systematically by varying attentional cues or load in a more continuous, parametric fashion. For instance, perhaps the contextual variation in mSPL activity in response to incongruent trials would be further enhanced under a stronger conflict frequency manipulation (e.g., if incongruent trials occurred only on $10 \%$ of trials in the low-conflict context or on $90 \%$ of trials in the high conflict context). In any event, the overall pattern of mSPL activation revealed here is broadly in line with the view that this region may be a source of a transient, domain-independent top-down control signal that initiates reconfiguration of the state of attentional selection in manner consistent with current contextual demands (Serences and Yantis, 2006; Yantis, 2008; Chiu and Yantis, 2009; Esterman et al., 2009; Greenberg et al., 2010; Tamber-Rosenau et al., 2011).

Consistent with this notion that mSPL activation reflects a top-down signal that resets the current state of attentional selection in a contextually appropriate manner, activation associated with contextual variation in conflict processing in this region covaried with activity in the early visual cortex and FFA ROIs. mSPL-mediated modulation of stimulus-driven sensory processing is commensurate with recent evidence demonstrating that disruption of $\mathrm{mSPL}$ activity with transcranial magnetic stimulation increases susceptibility to bottom-up attentional capture from irrelevant distracters (Kanai et al., 2011). Given the particular demands of our task on the visual system and the priming of control hypothesis, we assumed that the presentation of a stimulus in the high-conflict context would trigger top-down biasing of spatial processing in early visual cortex and/or target/distractor processing in the FFA. We found context-specific modulation of visual processing to be expressed as reduced lateralization in the FFA for stimuli presented in the high-conflict context. Although this finding echoes recent reports of spatially global gain modulation under conditions of increased cognitive control (Ester et al., 2009; King et al., 2010), the precise mechanisms by which this bilateral recruitment of the FFA for stimuli presented in the highconflict hemifield translated into context-specific improvements in interference resolution remain unclear.

Previous investigations of context-specific variation in interference resolution suggested that contextual cues prime retrieval of control settings in a rapid, online manner (Corballis and Gratton, 2003; Crump et al., 2006, 2008; Lehle and Hübner, 2008; Wendt et al., 2008; Vietze and Wendt, 2009; Wendt and Kiesel, 2011), but have not ruled out the alternative possibility that such effects might instead reflect a sustained focus of attention on the high-conflict context. The current finding that behavioral improvements in interference resolution were not immediately present on the first trial following transitions from the low- to high-conflict context speaks against a sustained allocation of attention on the high-conflict context. In contrast, the discovery that contextual variation in conflict processing in the mSPL was most pronounced upon context switches and that behavioral reduction in interference effects emerged only following repetitions of the high-conflict context suggests that control settings are rapidly retrieved upon exposure to the contextual cue and then facilitate performance on subsequent trials in that context.

The present results can be accommodated within the framework of a recent computational model (Verguts and Notebaert, 2008, 2009) developed to account for the phenomenon of context-specific [and item-specific (Blais et al., 2007)] improvements in interference resolution investigated here. According to the model, the experience of conflict elicits an arousal response (accompanied by noradrenalin release) that in turn promotes the binding of current bottom-up stimulus/context representations and top-down task representations/biasing processes via Hebbian (associative) learning. Applied to the current experiment, the model would explain the observed contextual variation in conflict processing as resulting from a binding process between the implicit contextual conflict-frequency cues and the associated top-down attentional control settings. This model can account not only for superior interference resolution in the high-conflict context by placing more emphasis on the task-relevant route when needed to overcome frequent incongruence, but also for inferior interference resolution in the low-conflict context by allowing usage of the easier, task-irrelevant route. A critical test of the applicability of the conflict-modulated Hebbian learning theory to findings such as those presented here would be to demonstrate contextual variation in autonomic indices of arousal.

To conclude, the current study delivers neural evidence substantiating the hypothesis that contextual cues can implicitly prime retrieval and implementation of top-down control settings. Following recent theoretical (Verguts and Notebaert, 2008, 2009) and empirical (Spapé and Hommel, 2008) work, we propose that top-down control settings associated with specific contextual information can be bound into episodic "event file" representations, and that, subsequently, their retrieval can be implicitly primed by other features of that representation. This challenges the traditional juxtaposition of bottom-up associative and 
top-down controlled processing and suggests that the two may in fact be inextricably linked.

\section{References}

Blais C, Robidoux S, Risko EF, Besner D (2007) Item-specific adaptation and the conflict-monitoring hypothesis: a computational model. Psychol Rev 114:1076-1086.

Blais C, Harris MB, Guerrero JV, Bunge SA (2012) Rethinking the role of automaticity in cognitive control. Q J Exp Psychol 65:268-276.

Botvinick MM, Braver TS, Barch DM, Carter CS, Cohen JD (2001) Conflict monitoring and cognitive control. Psychol Rev 108:624-652.

Boy F, Husain M, Sumner P (2010) Unconscious inhibition separates two forms of cognitive control. Proc Natl Acad Sci U S A 107:11134-11139.

Chiu YC, Yantis S (2009) A domain-independent source of cognitive control for task sets: shifting spatial attention and switching categorization rules. J Neurosci 29:3930-3938.

Corballis PM, Gratton G (2003) Independent control of processing strategies for different locations in the visual field. Biol Psychol 64:191-209.

Crump MJ, Milliken B (2009) The flexibility of context-specific control: evidence for context-driven generalization of item-specific control settings. Q J Exp Psychol 62:1523-1532.

Crump MJ, Gong Z, Milliken B (2006) The context-specific proportion congruent Stroop effect: location as a contextual cue. Psychon Bull Rev 13:316-321.

Crump MJ, Vaquero JM, Milliken B (2008) Context-specific learning and control: the roles of awareness, task relevance, and relative salience. Conscious Cogn 17:22-36.

Egner T (2007) Congruency sequence effects and cognitive control. Cogn Affect Behav Neurosci 7:380-390.

Egner T, Hirsch J (2005) Cognitive control mechanisms resolve conflict through cortical amplification of task-relevant information. Nat Neurosci 8:1784-1790.

Eriksen BA, Eriksen CW (1974) Effects of noise letters upon the identification of a target letter in a nonsearch task. Percept Psychophys 16:143-149.

Ester EF, Serences JT, Awh E (2009) Spatially global representations in human primary visual cortex during working memory maintenance. J Neurosci 29:15258-15265.

Esterman M, Chiu YC, Tamber-Rosenau BJ, Yantis S (2009) Decoding cognitive control in human parietal cortex. Proc Natl Acad Sci U S A 106:17974-17979.

Friston KJ, Buechel C, Fink GR, Morris J, Rolls E, Dolan RJ (1997) Psychophysiological and modulatory interactions in neuroimaging. Neuroimage 6:218-229.

Greenberg AS, Esterman M, Wilson D, Serences JT, Yantis S (2010) Control of spatial and feature-based attention in frontoparietal cortex. J Neurosci 30:14330-14339.

Heinemann A, Kunde W, Kiesel A (2009) Context-specific primecongruency effects: on the role of conscious stimulus representations for cognitive control. Conscious Cogn 18:966-976.

Hommel B (2004) Event files: feature binding in and across perception and action. Trends Cogn Sci 8:494-500.

Hommel B, Proctor RW, Vu KP (2004) A feature-integration account of sequential effects in the Simon task. Psychol Res 68:1-17.

Kanai R, Dong MY, Bahrami B, Rees G (2011) Distractibility in daily life is reflected in the structure and function of human parietal cortex. J Neurosci 31:6620-6626.

Kanwisher N, McDermott J, Chun MM (1997) The fusiform face area: a module in human extrastriate cortex specialized for face perception. J Neurosci 17:4302-4311.

Kelley TA, Lavie N (2011) Working memory load modulates distractor competition in primary visual cortex. Cereb Cortex 21:659-665.

King JA, Korb FM, von Cramon DY, Ullsperger M (2010) Post-error behavioral adjustments are facilitated by activation and suppression of task-relevant and task-irrelevant information processing. J Neurosci 30:12759-12769.

Lehle C, Hübner R (2008) On-the-fly adaptation of selectivity in the flanker task. Psychon Bull Rev 15:814-818.

Logan GD (1988) Toward an instance theory of automatization. Psychol Rev 95:492-527.

Mayr U, Awh E, Laurey P (2003) Conflict adaptation effects in the absence of executive control. Nat Neurosci 6:450-452.

Nee DE, Wager TD, Jonides J (2007) Interference resolution: insights from a meta-analysis of neuroimaging tasks. Cogn Affect Behav Neurosci 7:1-17.

Norman D, Shallice T (1986) Attention to action: willed and automatic control of behavior. In: Consciousness and self regulation: advances in research and theory (Davidson R, Schwartz G, Shapiro D, eds), pp 1-18. New York: Plenum.

Ridderinkhof KR, Ullsperger M, Crone EA, Nieuwenhuis S (2004) The role of the medial frontal cortex in cognitive control. Science 306:443-447.

Sarmiento BR, Shore DI, Milliken B, Sanabria D (2012) Audiovisual interactions depend on context of congruency. Atten Percept Psychophys 74:563-574

Schmidt JR, Crump MJ, Cheesman J, Besner D (2007) Contingency learning without awareness: evidence for implicit control. Conscious Cogn 16:421-435.

Schneider W, Shiffrin RM (1977) Controlled and automatic human information-processing. 1. Detection, search, and attention. Psychol Rev 84:1-66.

Serences JT, Schwarzbach J, Courtney SM, Golay X, Yantis S (2004) Control of object-based attention in human cortex. Cereb Cortex 14:1346-1357.

Serences JT, Yantis S (2006) Selective visual attention and perceptual coherence. Trends Cogn Sci 10:38-45.

Shiffrin RM, Schneider W (1977) Controlled and automatic human information processing. II. Perceptual learning, automatic attending, and a general theory. Psychol Rev 84:127-190.

Shulman GL, Astafiev SV, Franke D, Pope DL, Snyder AZ, McAvoy MP, Corbetta M (2009) Interaction of stimulus-driven reorienting and expectation in ventral and dorsal frontoparietal and basal ganglia-cortical networks. J Neurosci 29:4392-4407.

Spapé MM, Hommel B (2008) He said, she said: episodic retrieval induces conflict adaptation in an auditory Stroop task. Psychon Bull Rev 15:1117-1121.

Tamber-Rosenau BJ, Esterman M, Chiu YC, Yantis S (2011) Cortical mechanisms of cognitive control for shifting attention in vision and working memory. J Cogn Neurosci 23:2905-2919.

van Gaal S, Lamme VA, Ridderinkhof KR (2010) Unconsciously triggered conflict adaptation. PLoS One 5:e11508.

Vandenberghe R, Gitelman DR, Parrish TB, Mesulam MM (2001) Functional specificity of superior parietal mediation of spatial shifting. Neuroimage 14:661-673.

Verguts T, Notebaert W (2008) Hebbian learning of cognitive control: dealing with specific and nonspecific adaptation. Psychol Rev 115:518-525.

Verguts T, Notebaert W (2009) Adaptation by binding: a learning account of cognitive control. Trends Cogn Sci 13:252-257.

Vietze I, Wendt M (2009) Context specificity of conflict frequencydependent control. Q J Exp Psychol 62:1391-1400.

Wendt M, Kiesel A (2011) Conflict adaptation in time: foreperiods as contextual cues for attentional adjustment. Psychon Bull Rev 18:910-916.

Wendt M, Kluwe RH, Vietze I (2008) Location-specific versus hemispherespecific adaptation of processing selectivity. Psychon Bull Rev 15:135-140.

Yantis S (2008) The neural basis of selective attention: cortical sources and targets of attentional modulation. Curr Dir Psychol Sci 17:86-90.

Yantis S, Schwarzbach J, Serences JT, Carlson RL, Steinmetz MA, Pekar JJ, Courtney SM (2002) Transient neural activity in human parietal cortex during spatial attention shifts. Nat Neurosci 5:995-1002. 